\title{
Amniotic fluid stem cell extracellular vesicles promote fetal lung branching and cell differentiation in experimental congenital diaphragmatic hernia
}

Kasra Khalaj $^{1,2}$, Rebeca Lopes Figueira ${ }^{1,2}$, Lina Antounians ${ }^{1,2}$, Sree Gandhi ${ }^{1,2}$, Matthew Wales ${ }^{1,2}$, Louise Montalva $^{1,2}$, George Biouss ${ }^{1,2}$, and Augusto Zani ${ }^{1,2,3, *}$

${ }^{1}$ Developmental and Stem Cell Biology Program, Peter Gilgan Centre for Research and Learning, The Hospital for Sick Children, Toronto, Ontario, M5G 0A4, Canada.

${ }^{2}$ Division of General and Thoracic Surgery, The Hospital for Sick Children, Toronto, Ontario, M5G 1X8, Canada.

${ }^{3}$ Department of Surgery, University of Toronto, Toronto, M5T 1P5, Canada.

Running Title: Extracellular vesicles as therapy for CDH lungs

*Corresponding Author: Augusto Zani, MD, PhD, FACS, FAAP

Email: augusto.zani@sickkids.ca

Funding Sources: Canadian Institutes of Health Research (CIHR Project Grant 175300; AZ), CIHR Fellowship (176535, KK), American Thoracic Society (RP-2020-26; RLF), SickKids Restracomp Fellowship (KK), SickKids start-up funds (AZ), SickKids Foundation (R00DH00000, AZ), and University of Toronto Department of Surgery (AZ).

\section{Author contributions:}

Kasra Khalaj: Conception and design, collection and/or assembly of data, data analysis and interpretation, manuscript writing, final approval of manuscript

Rebeca Lopes Figueira: Collection and/or assembly of data, data analysis and interpretation, final approval of manuscript 
Lina Antounians: Collection and/or assembly of data, data analysis and interpretation, final approval of manuscript

Sree Gandhi: Collection and/or assembly of data, final approval of manuscript

Matthew Wales: Collection and/or assembly of data, final approval of manuscript

Louise Montalva: Collection and/or assembly of data, data analysis and interpretation, final approval of manuscript

George Biouss: Collection and/or assembly of data, final approval of manuscript

Augusto Zani: Conception and design, financial support, data analysis and interpretation, manuscript writing, final approval of manuscript

Key words: Exosome, EV, microRNA, CDH, pulmonary hypoplasia, lung development, fetal medicine, regenerative medicine

Data availability statement: The data that support the findings of this study are available from the corresponding author upon reasonable request. 


\section{ABSTRACT}

Pulmonary hypoplasia secondary to congenital diaphragmatic hernia $(\mathrm{CDH})$ is characterized by impaired branching morphogenesis and differentiation. We have previously demonstrated that administration of extracellular vesicles derived from rat amniotic fluid stem cells (AFSC-EVs) rescues development of hypoplastic lungs at the pseudoglandular and alveolar stages in rodent models of CDH. Herein, we tested whether AFSC-EVs exert their regenerative effects at the canalicular and saccular stages, as these are translationally relevant for clinical intervention. To induce fetal pulmonary hypoplasia, we gavaged rat dams with nitrofen at embryonic day 9.5 and demonstrated that nitrofen-exposed lungs had impaired branching morphogenesis, dysregulated signaling pathways relevant to lung development (FGF10/FGFR2, ROBO/SLIT, Ephrin, Neuropilin 1, $\beta$-catenin) and impaired epithelial and mesenchymal cell marker expression at both stages. AFSC-EVs administered to nitrofen-exposed lung explants rescued airspace density and increased the expression levels of key factors responsible for branching morphogenesis. Moreover, AFSC-EVs rescued the expression of alveolar type 1 and 2 cell markers at both canalicular and saccular stages, and restored markers of club, ciliated epithelial, and pulmonary neuroendocrine cells at the saccular stage. AFSC-EV treated lungs also had restored markers of lipofibroblasts and PDGFRA+ cells to control levels at both stages. EV tracking showed uptake of AFSC-EV RNA cargo throughout the fetal lung and an mRNA-miRNA network analysis identified that several miRNAs responsible for regulating lung development processes were contained in the AFSC-EV cargo. These findings suggest that AFSC-EV based therapies hold potential for restoring fetal lung growth and maturation in babies with pulmonary hypoplasia secondary to $\mathrm{CDH}$. 


\section{Introduction}

Fetal lung underdevelopment, also known as pulmonary hypoplasia, is primarily characterized by decreased lung growth and maturation [1]. The most common birth defect found in babies with pulmonary hypoplasia is congenital diaphragmatic hernia $(\mathrm{CDH}) . \mathrm{CDH}$ is characterized by incomplete closure of the fetal diaphragm and herniation of abdominal organs into the chest. At present, the mortality of $\mathrm{CDH}$ is $20-30 \%$ [2,3], and around $60 \%$ of survivors have long-term morbidity that may persist into adulthood [4]. Pulmonary hypoplasia is a primary cause of these poor outcomes, and it persists even after surgical repair of the diaphragm and repositioning of the herniated organs back into the abdomen [5]. The poor outcomes observed in infants and children with $\mathrm{CDH}$ have mobilized researchers to find an antenatal treatment that can promote normal lung development in fetuses with pulmonary hypoplasia secondary to $\mathrm{CDH}$ [6].

We have recently reported that administration of extracellular vesicles derived from rat amniotic fluid stem cells (AFSC-EVs) rescues normal development in rodent fetal models of pulmonary hypoplasia [7]. Specifically, we demonstrated that AFSC-EVs increase branching morphogenesis and promote epithelial cell differentiation in fetal rat lung explants harvested at the pseudoglandular stage [7]. Moreover, AFSC-EVs administered intra-tracheally in an in vivo rabbit fetal model of $\mathrm{CDH}$ increased the number of alveoli and promoted lipofibroblast differentiation at the alveolar stage of lung development [7]. The effects of AFSC-EVs on rat fetal lung growth and maturation were mainly mediated by their RNA cargo, as enzymatically digesting AFSC-EV RNA ablated their beneficial effects in multiple models [7]. Of the small 
RNA species contained in AFSC-EV cargo, miRNAs were the species most likely to mediate the regenerative effects observed on lung development [7].

When we previously investigated the biological effects of AFSC-EVs on fetal hypoplastic lungs, we reported that Fibroblast growth factor 10 (FGF10) expression was rescued to normal levels [7]. In that study, we had focused on the FGF10 pathway, as it plays a critical role in lung branching morphogenesis, epithelial proliferation, and lineage commitment [8]. However, fetal lung development is a highly dynamic process that requires multiple pathways to coordinate branching morphogenesis and lung cell differentiation [9]. Building on recent studies on the transcriptomic analysis and mapping of the rodent fetal lung [10,11], herein we investigated multiple signaling pathways that are known or unknown to be dysregulated in experimental $\mathrm{CDH}$. The findings reported in the present study expand on our knowledge of pulmonary hypoplasia and further elaborate on the impact of AFSC-EVs on regulatory pathways that are key for lung development. Moreover, we further delineated the effects of AFSC-EV therapy on hypoplastic lungs at the canalicular and saccular stages. As $\mathrm{CDH}$ is typically diagnosed at the anatomy scan around 20 weeks of gestation, these stages are translationally relevant and provide an opportunity for designing clinical interventional studies. Lastly, the present study further characterized the AFSC-EV effects on epithelial and mesenchymal cell differentiation, specifically interrogating some of the most relevant cell types during the canalicular and saccular stages of lung development. 


\section{Materials and Methods}

\section{Extracellular vesicle isolation and characterization}

Extracellular vesicles were derived from c-kit+ rat amniotic fluid stem cells (AFSCs) using our established protocol [12]. Briefly, AFSCs were grown to $70 \%$ confluency and the supernatant was collected and subjected to differential centrifugation (300 g, 1,200 g, and 100,000 g), as described [12]. Following the International Society for Extracellular Vesicles guidelines [13], EVs were characterized for size (nanoparticle tracking analysis), morphology (transmission electron microscopy), and expression of canonical EV-related protein markers (Western blot analysis), as described [7,12].

\section{Fluorescence labelling of AFSC-EVs and in vitro tracking}

AFSC-EVs were fluorescently labelled using ExoGlow-EV labeling kit (SBI System Biosciences), using the manufacturer's protocol. The fluorescently labelled AFSC-EVs were added to E17.5 and E20.5 fetal rat explant cultures and co-incubated with 4',6-diamidino-2phenylindole (DAPI) for $3 \mathrm{hrs}$ prior to imaging, using a 2-photon Leica SP8 confocal microscope (Leica).

\section{Experimental models of pulmonary hypoplasia}

Ex vivo - In fetal rats, $\mathrm{CDH}$ and pulmonary hypoplasia were induced via the administration of nitrofen to rat dams (100 mg) by oral gavage on embryonic day (E) 9.5. Dams were euthanized and fetal lungs were micro-dissected at the canalicular (E17.5) and saccular (E20.5) stages of lung development. Lungs were grown as explants on nanopore membranes (Whatman, Thermofisher Scientific), and incubated for $72 \mathrm{~h}$ in culture medium alone or rat AFSC-EVs 
$(0.5 \% \mathrm{v} / \mathrm{v})$. Lungs from fetuses of dams that received olive oil (no nitrofen) at E9.5 served as control. All explant specimens were snap frozen in liquid nitrogen and stored at $-80{ }^{\circ} \mathrm{C}$ for longterm storage or fixed and processed for histology using our established protocol [7].

In vitro - Primary fibroblasts were derived from fetal rat lungs dissected at E18.5 using an established protocol [14]. Briefly, single cell suspensions were obtained from pooled lungs of control and nitrofen-exposed rat fetuses by trypsinization ( $0.25 \%$ trypsin; Gibco). Fibroblasts were isolated as described $[7,14,15]$, grown for 7 days and then treated with culture medium alone or rat AFSC-EVs $(0.5 \% \mathrm{v} / \mathrm{v})$ for $24 \mathrm{~h}$. Upon harvest, fibroblast gene expression analysis was conducted.

\section{Branching morphogenesis evaluation}

To assess branching, rat fetal lung explants were processed for histology (hematoxylin/eosin staining) as described [7]. Explants were evaluated for airspace density using the radial airspace count (RAC) and parenchymal to airspace ratio using the mean linear intercept (MLI), as recommended by the American Thoracic Society [16].

\section{mRNA quantification with $q R T-P C R$}

RNA extraction was conducted using Trizol and cDNA synthesis was performed using the VILO superscript RT III kit (Life Technologies), as described [7]. Gene expression was conducted using the $2^{\wedge}$-deltadeltaCT method using ViiA qPCR system (ABI) following MIQE guidelines [17]. Primers are listed in Table 1.

\section{Protein extraction and Western blotting}


Protein extraction and Western blotting were performed, as previously described [7]. Briefly, cells or tissues were subjected to tissue extraction buffer (ThermoFisher Scientific) containing protease and phosphatase inhibitor cocktail (Roche) and disrupted by sonication. The protein content was quantified using a bicinchoninic acid assay (ThermoFisher Scientific), normalized to $10 \mu \mathrm{g}$, and loaded on 4-12\% NuPage MES mini-blot pre-cast gels (ThermoFisher Scientific). Transfer was performed using the iBlot2 system (ThermoFisher Scientific) and imaged using an Odyssey FC digital acquisition system (LI-COR Biosciences). Antibodies and concentrations are specified in Table 2 .

\section{Immunofluorescence using confocal microscopy}

Immunofluorescence was conducted using our established protocol [7]. Paraffin-embedded sections were stained using the following antibodies: Podoplanin (PDPN) for alveolar type 1 (AT1) cells, Surfactant protein C (SPC) for alveolar type 2 (AT2) cells, both co-stained with Epithelial adherens junction adherin (ECAD); Club-cell-specific $10 \mathrm{kD}$ protein (CC10) for club cells; Tumor protein 63 (P63) for basal cells; Forkhead box protein J1 (FOXJ1) for ciliated epithelial cells; Achaete-scute homolog 1 (ASCL1) for pulmonary neuroendocrine cells; Plateletderived growth factor receptor A (PDGFRA) and Peroxisome proliferator-activated receptor gamma (PPARG) for mesenchymal cells. Antibodies and concentrations are provided in Table 2.

\section{Results}

Administration of AFSC-EVs rescues branching morphogenesis in fetal hypoplastic lungs during the canalicular and saccular stages of lung development 
We first confirmed that nitrofen-exposed lungs harvested at both canalicular and saccular stages of lung development had impaired branching morphogenesis, as evidenced by fewer airspaces measured with RAC and a lower airspace to parenchyma ratio measured by MLI (Figure 1A-B). Administration of AFSC-EVs to nitrofen-exposed lung explants rescued branching morphogenesis at both developmental stages. When we investigated signaling pathways that are known to be responsible for branching morphogenesis [8], we found that nitrofen-exposed lungs harvested at either E17.5 or E20.5 had downregulated expression of Fgf10, Roundabout guidance receptor 2 (Robo2), and Slit guidance ligand 2 (Slit2) factors, as well as upregulated expression of Roundabout guidance receptor 1 (Robol) (Figure 1C-D). Moreover, fetal lungs harvested at E20.5 had higher levels of Fibroblast growth factor receptor 2 (Fgfr2) and lower levels of Ephrin B2 (Efnb2), Ephrin type-B receptor 2 (Ephb2), Neuropilin 1 (Nrp1), and $\beta$ catenin 1 (Cttnb1) compared to control (Figure 1D). Interestingly, nitrofen-exposed lungs did not have any dysregulation of other factors that are also involved in branching morphogenesis [8], such as Wnt family member 3a and 5a (Wnt3a and Wnt5a), Semaphorin 3a (Sema3a), and Plexin A (Plxna) at any of the timepoints (Supplementary Figure 1). Following AFSC-EV administration, nitrofen-exposed lungs harvested at E17.5 or E20.5 had upregulated levels of Fgf10, Robo2, and Slit2 and downregulated levels of Fgfr2 and Robo1. Moreover, AFSC-EV treated hypoplastic lungs harvested at E20.5 had upregulated levels of Efnb2, Ephb2, and Nrp1 (Figure 1C-D).

\section{AFSC-EV administration promotes differentiation of epithelial lung cells at canalicular and saccular stages of lung development}


Nitrofen-exposed lungs have been reported to be immature, in part due to the altered differentiation of the proximal and distal airway epithelium. At the canalicular stage, we observed that nitrofen exposure decreased the expression of markers for AT1 (PDPN) and AT2 (SPC) cells but had no effect on markers of club cells (CC10), basal cells (P63), ciliated epithelial cells (FOXJ1), and pulmonary neuroendocrine cells (ASCL1) (Figure 2A-C, Supplementary Figure 2). Administration of AFSC-EVs to nitrofen-exposed lungs harvested at the canalicular stage rescued the expression levels of AT1 and AT2 cell markers back to control.

At the saccular stage, we observed a dysregulation of expression markers of proximal and distal epithelial cells (Figure 3A-C). Nitrofen-exposed lungs harvested at this stage and treated with AFSC-EVs had increased expression of AT1, AT2, club, and ciliated epithelial cells, and decreased expression of pulmonary neuroendocrine cells back to control (Figure 3A-C).

\section{AFSC-EV administration has regenerative effects on the fetal lung mesenchyme}

Several studies have reported that $\mathrm{CDH}$ hypoplastic lungs have undifferentiated mesenchyme $[18,19]$, with altered expression of lipofibroblasts [20-23] and PDGFRA+ cells [24,25]. To study mesenchymal differentiation and lung fibroblast response to AFSC-EVs, we first derived primary fibroblasts from control and nitrofen-exposed lungs harvested at the canalicular stage. In primary cell culture, we observed a decreased expression of PPARG and Thy1, which are markers of lipofibroblasts [26]. Moreover, we found an increase in PDGFRA expression, which is a marker of progenitor cells that mainly give rise to myofibroblasts [27], but no difference in expression of Actin alpha 2 (Acta2), a well-established marker of myofibroblasts [18,19]. When we investigated the mesenchyme of fetal lung explants, we confirmed the decrease in 
lipofibroblast markers at both gene and protein expression levels in nitrofen-exposed fetal lungs harvested at canalicular (Figure 4B-D) and saccular stages (Figure 4E-G). The increase in PDGFRA+ cell expression noticed in primary fibroblasts derived from nitrofen-exposed lungs was also confirmed in the explant model at the canalicular stage (Figure 4B-D). Conversely, the protein expression of PDGFRA in fetal nitrofen-exposed lungs harvested at the saccular stage was decreased compared to control (Figure 4F-G). Administration of AFSC-EVs to nitrofenexposed primary fibroblasts or lung explants increased the expression of lipofibroblast markers (Figure 4A-G). Similarly, nitrofen-exposed primary fibroblasts and fetal lungs treated with AFSC-EVs had a decrease in PDGFRA expression levels compared to nitrofen-injured untreated fibroblasts or lung explants (Figure 4B-G).

\section{AFSC-EV mechanism of action is partly modulated by the release of RNA species}

As we have previously demonstrated that AFSC-EV main mechanism of action towards fetal lung regeneration at E14.5 is through the release of the RNA cargo [7], in this study we tracked the RNA cargo of AFSC-EVs that were administered to nitrofen-exposed lungs harvested at E17.5 and E20.5. At both stages of lung development, we observed that AFSC-EVs entered the lung tissue, as shown by the presence of their RNA cargo throughout the lung parenchyma (Figure 5A-B, Supplementary online Video 1). In our previous small RNA sequencing studies [7], we identified some miRNAs that were in part responsible for the effects of AFSC-EVs on branching morphogenesis and fetal lung epithelial differentiation. In the present study, we linked the genes responsible for branching morphogenesis and cell differentiation that were downregulated following AFSC-EV treatment (Robol, Fgfr2, Ascll, and Pdgfra; Figures 1,3,4) 
with miRNAs that were found to be in the AFSC-EV cargo by the small RNA sequencing analysis conducted in our previous study (Figure 5C). Using miRWalk, we found 91 miRNAs that are predicted to downregulate the selected 4 genes, including 28 that are known to be involved in lung development (Figure 5C). Of the 91 miRNAs, we identified 11 miRNAs that co-regulated more than one gene.

\section{Discussion}

In this study, we have shown that AFSC-EVs administered to rat fetal hypoplastic lungs harvested at canalicular and saccular stages of lung development exert a regenerative effect on the pulmonary epithelium and mesenchyme. These effects were through multiple pathways that are known to be involved in lung development [8] and that we herein demonstrated to be targeted by AFSC-EVs. When we investigated the FGF pathway in the nitrofen model, we replicated the observation of other research groups that reported a consistent decrease in FGF10 expression at both canalicular and saccular stages [28-32], and an upregulation of FGFR2 expression at the saccular stage [33]. As we had previously demonstrated in fetal lung explants harvested at the pseudoglandular stage [7], AFSC-EVs administered to explants harvested at both canalicular and saccular stages rescued the expression of both FGF10 and FGFR2 back to control levels. The ROBO/SLIT pathway is also essential for normal lung development, as shown in knock-out studies [34,35], and is downregulated in nitrofen-exposed lungs of fetuses with CDH [36]. In the present study, we confirmed the upregulation of ROBO1 and the corresponding downregulation of ROBO2 and SLIT2 expression in nitrofen-exposed lungs at both timepoints, which were rescued by AFSC-EV treatment to control levels. When we assessed factors involved in sending guidance cues for lung branching, we confirmed that the Ephrin pathway was downregulated at 
the saccular stage, as previously reported [37], and rescued by AFSC-EV treatment to control levels. Neuropilin is another guidance cue factor that has been reported to support the formation of alveolar ducts and alveoli in rodents [38] and that, to the best of our knowledge, has not been investigated in nitrofen-exposed lungs. The present study shows for the first time that Neuropilin gene expression is downregulated in nitrofen-exposed lungs during the saccular stage and rescued by AFSC-EV administration. Similarly, $\beta$-Catenin, which is important for the maintenance of lung epithelial progenitors [39], is herein reported for the first time to have downregulated expression in nitrofen-exposed lungs. However, treatment with AFSC-EVs did not affect $\beta$-Catenin gene expression levels. Lastly, when we investigated other factors implicated in lung branching morphogenesis, such as Wnt family members WNT3A and WNT5A, as well as Semaphorin, and Plexin A [8], we found no differences between control and nitrofen-exposed lungs. These results support the notion that some but not all of the pathways involved in branching morphogenesis are dysregulated in human and experimental CDH.

In this study, we confirmed that fetal hypoplastic lungs have impaired epithelial cell differentiation, as we had reported at the pseudoglandular stage [7]. In the distal airways, we found reduced expression of AT1 and AT2 markers at both canalicular and saccular stages of lung development, as previously reported by other groups [25,40-42]. AT1 and AT2 downregulation was rescued by AFSC-EV administration to control levels, in line with our previous report in fetal lungs harvested at the pseudoglandular stage [7]. When we investigated epithelial cell populations in the proximal airways, we observed no differences in the expression of club, basal, and ciliated epithelial cells at the canalicular stage, similar to reports from human and rodent models of pulmonary hypoplasia at the same timepoint $[25,43]$. At the saccular stage, 
we observed downregulated expression of club and ciliated epithelial cell markers, as well as upregulated expression of pulmonary neuroendocrine cells in nitrofen-exposed lungs harvested. To the best of our knowledge, no study investigated the expression of basal, club, and ciliated epithelial cells in nitrofen-exposed lungs at the saccular stage. Conversely, we confirmed the upregulation of pulmonary neuroendocrine cells at the saccular stage of lung development, as shown by several groups [44-46]. The increased number of pulmonary neuroendocrine cells in $\mathrm{CDH}$ fetal lungs has previously been explained as an adaptive response to impaired lung development, whereby the amines and peptides secreted by these cells are implicated in lung development, repair, and regeneration $[44,47]$. The dysregulated expression of epithelial cell populations lining the proximal airway were restored to normal levels by AFSC-EV administration at both canalicular and saccular stages.

The mesenchyme plays a critical role in lung development, as it provides support for the fetal lung epithelium [48-50]. In this study, we confirmed that hypoplastic CDH lungs have decreased expression of lipofibroblasts, as previously shown in the nitrofen model [19-21,23] and in the surgical rabbit model of $\mathrm{CDH}$ [7]. Lipofibroblasts, a population of lipid-containing fibroblasts that develop during the canalicular stage, play a crucial role during fetal lung development, maintain tissue homeostasis, and respond to cellular injury [48,51]. PDGFRA+ cells are a population of progenitors that mainly give rise to myofibroblasts [27]. This cell population is key for lung development and its conditional inactivation during embryogenesis has been reported to result in alveolar simplification [27]. In our study, we found increased levels of PDGFRA in vitro and ex vivo at the canalicular stage, as previously reported during the same developmental stage by Dingemann et al [24]. The alteration in markers of lipofibroblasts and PDGFRA+ cells 
in nitrofen-exposed lungs compared to control could indicate that hypoplastic fetal lungs remain at an immature state, with decreased cell differentiation and increased levels of progenitor cells. This is in line with other studies reporting that the pulmonary mesenchyme is defective in the nitrofen model of pulmonary hypoplasia and may be due to the dysregulated epithelialmesenchymal tissue interactions $[18,42]$. The decrease in lipofibroblast markers observed in this study at the canalicular stage persisted also in the saccular stage of lung development. Conversely, we observed that PDGFRA protein expression was decreased in nitrofen-exposed lungs compared to control at the saccular stage. This could be due to simplification of the lung architecture, which is also observed in immature lungs affected by bronchopulmonary dysplasia, where PDGFRA expression has been reported to be downregulated [52-54]. In both canalicular and saccular stages of lung development, AFSC-EV administration to nitrofen-exposed lungs restored markers of lipofibroblasts and PDGFRA+ cells back to control levels. These findings in the mesenchyme were similar to the improvements observed in lung epithelial differentiation. Given the close interaction between epithelium and mesenchyme compartments during lung development, it is difficult to delineate whether EV effects are directed toward one compartment and affects the other indirectly or are affecting both simultaneously.

The EV-tracking experiments conducted in this and our previous study [7] demonstrate that the AFSC-EV cargo uptake is not specific to an area or compartment of the lung. Studies investigating potential cell-type specific transcriptomic changes are currently underway. Nonetheless, in the present study, we were able to find regulators of both lung epithelial and mesenchymal cells. In fact, our analysis of the AFSC-EV miRNA cargo and downregulated genes revealed 28 miRNAs that play a role in restored epithelial and mesenchymal cell 
differentiation, as well as branching morphogenesis. As an example, miR-20-5p, which belongs to the miR17 92 family, is of particular interest as it controls pulmonary surfactant gene expression in AT2 cells [55]. In addition to this cluster, miR-28-5p was also found in our network to regulate Robol and Pdgfra, and has been reported to be decreased in the nitrofen model of $\mathrm{CDH}[7,56]$. Interestingly, 11 miRNAs were identified in our network to regulate more than one of the target genes, indicating that target gene regulation can be coordinated by multiple combinations of miRNAs [57]. Together, these findings support that AFSC-EV mechanism of action in restoring tissue homeostasis is partly ascribed to their RNA cargo transfer.

This study provides further evidence that an AFSC-EV-based therapy holds potential for fetal lung regeneration in babies with pulmonary hypoplasia secondary to $\mathrm{CDH}$. We have herein demonstrated that AFSC-EVs promote branching morphogenesis and rescue epithelial and mesenchymal cell homeostasis at both canalicular and saccular stages of lung development. Towards the translational advancement of this novel therapy, these timepoints are amenable for fetal intervention, especially in fetuses with $\mathrm{CDH}$ who are diagnosed prior to these stages. We specifically found that while the changes in differentiation of the distal airways occurred in both the canalicular and saccular stages, the proximal epithelium was rescued only in the saccular stage. However, it is difficult to translate these findings from a rodent model directly to human babies. Fetal interventions in human babies with $\mathrm{CDH}$, such as fetal endoscopic tracheal occlusion, have been reported during both time points [58,59]. Future studies will examine the optimal gestational age to administer the EV-therapy and will address other aspects such as route of delivery and dosage in larger animal models. In fact, it is critical to carefully address these and 
other translational aspects before clinical application of EV-based therapies in human patients, as previously reported $[60,61]$.

We acknowledge that our study has some limitations. Our findings were obtained using the nitrofen model of $\mathrm{CDH}$, which is based on the administration of an herbicide that targets retinoic acid synthesis $[26,62]$. Nonetheless, although human pulmonary hypoplasia is not caused by maternal exposure to herbicides, nitrofen administration causes a degree of lung underdevelopment analogous to that of human fetuses with pulmonary hypoplasia $[26,62]$. Moreover, retinoic acid is known to play a role in fetal lung development and CDH babies have low intracellular retinoic acid levels in the lungs and low retinol and retinol-binding protein levels in the cord blood $[63,64]$. This model has been used for over three decades and the multiple pathways that are dysregulated in nitrofen-exposed hypoplastic lungs are similar to those reported in human studies [26]. Nevertheless, findings from our study serve mainly as a proof-of-principle that AFSC-EVs rescue lung development in $\mathrm{CDH}$ hypoplastic lungs. Moreover, we recognize that our study addressed the epithelial and mesenchymal compartments of the lung, for what is currently known. While there is a vast literature on epithelial differentiation in normal and hypoplastic lungs, relatively little is known about lung mesenchymal cells and their impairment in pulmonary hypoplasia. This is in line with a recent report by Tsukui et al, who highlighted that the diversity of lung fibroblasts has not been well characterized in normal or diseased lungs [50]. Nonetheless, we are encouraged by the observation that AFSC-EVs are able to promote differentiation towards lipofibroblast in canalicular and saccular stages in rats, as well as during alveologenesis in fetal rabbits with $\mathrm{CDH}$ [7]. 
In conclusion, this study confirms that administration of AFSC-EVs to hypoplastic fetal lungs rescues branching morphogenesis and promotes differentiation of epithelial and mesenchymal lung cells during the canalicular and saccular stages of lung development. Moreover, we provide further evidence that the RNA cargo could be responsible for the AFSC-EV mechanism of action towards lung regeneration. Taken together, these findings demonstrate that AFSC-EV treatment may represent an exciting new opportunity for antenatal intervention for CDH babies at clinically relevant timepoints.

\section{Acknowledgements}

The authors would like to thank Rachel Bercovitch for technical assistance and Paolo De Coppi for providing AFSCs in kind. We are indebted to the Imaging Facility and the Lab Animal Services at the Hospital for Sick Children, Toronto. The graphical abstract was designed using BioRender.com.

\section{Conflict of interest}

The authors declared no potential conflicts of interest. 


\section{References}

1 Cotten CM. Pulmonary hypoplasia. Semin Fetal Neonatal Med 2017;22:250-255.

2 Harting MT, Lally KP. The Congenital Diaphragmatic Hernia Study Group registry update. Semin Fetal Neonatal Med 2014;19:370-375.

3 Gupta VS, Harting MT, Lally PA, et al. Mortality in Congenital Diaphragmatic Hernia: A Multicenter Registry Study of Over 5000 Patients Over 25 Years. Ann Surg 2021.

4 Spoel M, van der Cammen-van Zijp MHM, Hop WCJ, et al. Lung function in young adults with congenital diaphragmatic hernia; a longitudinal evaluation. Pediatr Pulmonol 2013;48:130-137.

5 Hislop A, Reid L. Persistent hypoplasia of the lung after repair of congenital diaphragmatic hernia. Thorax 1976;31:450-455.

6 Jeanty C, Kunisaki SM, MacKenzie TC. Novel non-surgical prenatal approaches to treating congenital diaphragmatic hernia. Semin Fetal Neonatal Med 2014;19:349-356.

7 Antounians L, Catania VD, Montalva L, et al. Fetal lung underdevelopment is rescued by administration of amniotic fluid stem cell extracellular vesicles in rodents. Sci Transl Med 2021;13:eaax5941.

8 Horowitz A, Simons M. Branching morphogenesis. Circ Res 2008;103:784-795.

9 Herriges M, Morrisey EE. Lung development: orchestrating the generation and regeneration of a complex organ. Development 2014;141:502-513.

10 Guo M, Du Y, Gokey JJ, et al. Single cell RNA analysis identifies cellular heterogeneity 
and adaptive responses of the lung at birth. Nat Commun 2019;10:37.

11 Ardini-Poleske ME, Clark RF, Ansong C, et al. LungMAP: The Molecular Atlas of Lung Development Program. Am J Physiol Lung Cell Mol Physiol 2017;313:L733-L740.

12 Antounians L, Tzanetakis A, Pellerito O, et al. The Regenerative Potential of Amniotic Fluid Stem Cell Extracellular Vesicles: Lessons Learned by Comparing Different Isolation Techniques. Sci Rep 2019;9:1837.

13 Thery C, Witwer KW, Aikawa E, et al. Minimal information for studies of extracellular vesicles 2018 (MISEV2018): a position statement of the International Society for Extracellular Vesicles and update of the MISEV2014 guidelines. J Extracell Vesicles 2018;7:1535750.

14 Caniggia I, Tseu I, Han RN, et al. Spatial and temporal differences in fibroblast behavior in fetal rat lung. Am J Physiol 1991;261:L424-33.

15 Tzanetakis A, Antounians L, Belfiore A, et al. Endoplasmic reticulum stress response is activated in pulmonary hypoplasia secondary to congenital diaphragmatic hernia, but is decreased by administration of amniotic fluid stem cells. Pediatr Surg Int 2019;35:63-69.

16 Hsia CCW, Hyde DM, Ochs M, et al. An official research policy statement of the American Thoracic Society/European Respiratory Society: standards for quantitative assessment of lung structure. Am J Respir Crit Care Med 2010;181:394-418.

17 Bustin SA, Benes V, Garson JA, et al. The MIQE guidelines: minimum information for publication of quantitative real-time PCR experiments. Clin Chem 2009;55:611-622.

18 van Loenhout RB, Tseu I, Fox EK, et al. The pulmonary mesenchymal tissue layer is 
defective in an in vitro recombinant model of nitrofen-induced lung hypoplasia. Am $\mathbf{J}$ Pathol 2012;180:48-60.

19 Donahoe PK, Longoni M, High FA. Polygenic Causes of Congenital Diaphragmatic Hernia Produce Common Lung Pathologies. Am J Pathol 2016;186:2532-2543.

20 Friedmacher F, Hofmann AD, Takahashi H, et al. Disruption of THY-1 signaling in alveolar lipofibroblasts in experimentally induced congenital diaphragmatic hernia. Pediatr Surg Int 2014;30:129-135.

21 Friedmacher F, Fujiwara N, Hofmann AD, et al. Evidence for decreased lipofibroblast expression in hypoplastic rat lungs with congenital diaphragmatic hernia. Pediatr Surg Int 2014;30:1023-1029.

22 Gosemann J-H, Doi T, Kutasy B, et al. Alterations of peroxisome proliferator-activated receptor $\gamma$ and monocyte chemoattractant protein 1 gene expression in the nitrofeninduced hypoplastic lung. J Pediatr Surg 2012;47:847-851.

23 Friedmacher F, Fujiwara N, Hofmann AD, et al. Prenatal retinoic acid increases lipofibroblast expression in hypoplastic rat lungs with experimental congenital diaphragmatic hernia. J Pediatr Surg 2014;49:876-881.

24 Dingemann J, Doi T, Ruttenstock E, et al. Abnormal platelet-derived growth factor signaling accounting for lung hypoplasia in experimental congenital diaphragmatic hernia. J Pediatr Surg 2010;45:1989-1994.

25 Kunisaki SM, Jiang G, Biancotti JC, et al. Human induced pluripotent stem cell-derived lung organoids in an ex $\square$ vivo model of the congenital diaphragmatic hernia fetal lung. 
Stem Cells Transl Med 2021;10:98-114.

26 Montalva L, Zani A. Assessment of the nitrofen model of congenital diaphragmatic hernia and of the dysregulated factors involved in pulmonary hypoplasia. Pediatr Surg Int 2019;35:41-61.

27 Li R, Bernau K, Sandbo N, et al. Pdgfra marks a cellular lineage with distinct contributions to myofibroblasts in lung maturation and injury response. Elife 2018;7.

28 Teramoto H, Yoneda A, Puri P. Gene expression of fibroblast growth factors 10 and 7 is downregulated in the lung of nitrofen-induced diaphragmatic hernia in rats. J Pediatr Surg 2003;38:1021-1024.

29 Acosta JM, Thébaud B, Castillo C, et al. Novel mechanisms in murine nitrofen-induced pulmonary hypoplasia: FGF-10 rescue in culture. Am J Physiol Lung Cell Mol Physiol 2001;281:L250-7.

30 Chalphin A V, Tracy SA, Lazow SP, et al. Congenital diaphragmatic hernia as a potential target for transamniotic stem cell therapy. J Pediatr Surg 2020;55:249-252.

31 Pederiva F, Ghionzoli M, Pierro A, et al. Amniotic fluid stem cells rescue both in vitro and in vivo growth, innervation, and motility in nitrofen-exposed hypoplastic rat lungs through paracrine effects. Cell Transplant 2013;22:1683-1694.

32 Jesudason EC, Smith NP, Connell MG, et al. Peristalsis of airway smooth muscle is developmentally regulated and uncoupled from hypoplastic lung growth. Am J Physiol Lung Cell Mol Physiol 2006;291:L559-65.

33 Friedmacher F, Doi T, Gosemann J-H, et al. Upregulation of fibroblast growth factor 
receptor 2 and 3 in the late stages of fetal lung development in the nitrofen rat model.

Pediatr Surg Int 2012;28:195-199.

34 Xian J, Clark KJ, Fordham R, et al. Inadequate lung development and bronchial hyperplasia in mice with a targeted deletion in the Dutt1/Robol gene. Proc Natl Acad Sci U S A 2001;98:15062-15066.

35 Greenberg JM, Thompson FY, Brooks SK, et al. Slit and robo expression in the developing mouse lung. Dev Dyn 2004;230:350-360.

36 Gonçalves AN, Correia-Pinto J, Nogueira-Silva C. ROBO2 signaling in lung development regulates SOX2/SOX9 balance, branching morphogenesis and is dysregulated in nitrofen-induced congenital diaphragmatic hernia. Respir Res 2020;21:302.

37 Takahashi T, Friedmacher F, Zimmer J, et al. Ephrin-B1, -B2, and -B4 Expression is Decreased in Developing Diaphragms and Lungs of Fetal Rats with Nitrofen-Induced Congenital Diaphragmatic Hernia. Eur J Pediatr Surg 2019;29:113-119.

38 McGowan SE, McCoy DM. Neuropilin-1 directs PDGFR $\alpha$-entry into lung fibroblasts and signaling from very early endosomes. Am J Physiol Lung Cell Mol Physiol 2021;320:L179-L192.

39 Ostrin EJ, Little DR, Gerner-Mauro KN, et al. $\beta$-Catenin maintains lung epithelial progenitors after lung specification. Development 2018;145.

40 Nguyen TM, Jimenez J, Rendin LE, et al. The proportion of alveolar type 1 cells decreases in murine hypoplastic congenital diaphragmatic hernia lungs. PLoS One 
2019;14:e214793.

41 Chapin CJ, Ertsey R, Yoshizawa J, et al. Congenital diaphragmatic hernia, tracheal occlusion, thyroid transcription factor-1, and fetal pulmonary epithelial maturation. Am $\mathbf{J}$ Physiol Lung Cell Mol Physiol 2005;289:L44-52.

42 Guilbert TW, Gebb SA, Shannon JM. Lung hypoplasia in the nitrofen model of congenital diaphragmatic hernia occurs early in development. Am J Physiol Lung Cell Mol Physiol 2000;279:L1159-71.

43 Serapiglia V, Stephens C, Joshi R, et al. Murine Fetal Tracheal Occlusion Increases Lung Basal Cells via Increased Yap Signaling. MedRxiv 2021:2021.09.17.21263741.

44 Gosney JR, Okoye BO, Lloyd DA, et al. Pulmonary neuroendocrine cells in nitrofeninduced diaphragmatic hernia and the effect of prenatal glucocorticoids. Pediatr Surg Int 1999;15:180-183.

45 Santos M, Nogueira-Silva C, Baptista MJ, et al. Pulmonary epithelial cell differentiation in the nitrofen-induced congenital diaphragmatic hernia. J Pediatr Surg 2007;42:12311237.

46 IJsselstijn H, Perrin DG, de Jongste JC, et al. Pulmonary neuroendocrine cells in neonatal rats with congenital diaphragmatic hernia. J Pediatr Surg 1995;30:413-415.

47 Song H, Yao E, Lin C, et al. Functional characterization of pulmonary neuroendocrine cells in lung development, injury, and tumorigenesis. Proc Natl Acad Sci U S A 2012;109:17531-17536.

48 Riccetti M, Gokey JJ, Aronow B, et al. The elephant in the lung: Integrating lineage- 
tracing, molecular markers, and single cell sequencing data to identify distinct fibroblast populations during lung development and regeneration. Matrix Biol 2020;91-92:51-74.

49 El Agha E, Moiseenko A, Kheirollahi V, et al. Two-Way Conversion between Lipogenic and Myogenic Fibroblastic Phenotypes Marks the Progression and Resolution of Lung Fibrosis. Cell Stem Cell 2017;20:261-273.e3.

50 Tsukui T, Sun K-H, Wetter JB, et al. Collagen-producing lung cell atlas identifies multiple subsets with distinct localization and relevance to fibrosis. Nat Commun 2020;11:1920.

51 Rehan VK, Sugano S, Wang Y, et al. Evidence for the presence of lipofibroblasts in human lung. Exp Lung Res 2006;32:379-393.

52 Popova AP, Bentley JK, Cui TX, et al. Reduced platelet-derived growth factor receptor expression is a primary feature of human bronchopulmonary dysplasia. Am J Physiol Lung Cell Mol Physiol 2014;307:L231-9.

53 Fulton CT, Cui TX, Goldsmith AM, et al. Gene Expression Signatures Point to a Male Sex-Specific Lung Mesenchymal Cell PDGF Receptor Signaling Defect in Infants Developing Bronchopulmonary Dysplasia. Sci Rep 2018;8:17070.

54 Li C, Lee MK, Gao F, et al. Secondary crest myofibroblast PDGFR $\alpha$ controls the elastogenesis pathway via a secondary tier of signaling networks during alveologenesis. Development 2019;146.

55 Gong $\mathrm{Y}, \mathrm{Xu} \mathrm{W}$, Chen $\mathrm{Y}$, et al. miR-20a-5p regulates pulmonary surfactant gene expression in alveolar type II cells. J Cell Mol Med 2019;23:7664-7672. 
56 Zhu S, He Q, Zhang R, et al. Decreased expression of miR-33 in fetal lungs of nitrofeninduced congenital diaphragmatic hernia rat model. J Pediatr Surg 2016;51:1096-1100.

57 Mukherji S, Ebert MS, Zheng GXY, et al. MicroRNAs can generate thresholds in target gene expression. Nat Genet 2011;43:854-859.

58 Deprest JA, Nicolaides KH, Benachi A, et al. Randomized Trial of Fetal Surgery for Severe Left Diaphragmatic Hernia. N Engl J Med 2021;385:107-118.

59 Deprest JA, Benachi A, Gratacos E, et al. Randomized Trial of Fetal Surgery for Moderate Left Diaphragmatic Hernia. N Engl J Med 2021;385:119-129.

60 Rohde E, Pachler K, Gimona M. Manufacturing and characterization of extracellular vesicles from umbilical cord-derived mesenchymal stromal cells for clinical testing. Cytotherapy 2019;21:581-592.

61 Khalaj K, Figueira RL, Antounians L, et al. Systematic review of extracellular vesiclebased treatments for lung injury: are EVs a potential therapy for COVID-19? J Extracell Vesicles 2020;9:1795365.

62 Antounians L, Figueira RL, Sbragia L, et al. Congenital Diaphragmatic Hernia: State of the Art in Translating Experimental Research to the Bedside. Eur J Pediatr Surg 2019;29:317-327.

63 Coste K, Beurskens LWJE, Blanc P, et al. Metabolic disturbances of the vitamin A pathway in human diaphragmatic hernia. Am J Physiol Lung Cell Mol Physiol 2015;308:L147-57.

64 Loo CKC, Pearen MA, Pereira TN, et al. Lung and liver growth and retinoic acid status 
bioRxiv preprint doi: https://doi.org/10.1101/2022.01.10.475632; this version posted January 12,2022 . The copyright holder for this preprint (which was not certified by peer review) is the author/funder, who has granted bioRxiv a license to display the preprint in perpetuity. It is made available under aCC-BY-NC-ND 4.0 International license.

in human fetuses with congenital diaphragmatic hernia. Early Hum Dev 2018;116:17-

23. 


\section{Figure Legends}

Figure 1. Administration of AFSC-EVs rescues branching morphogenesis in fetal hypoplastic lungs during the canalicular and saccular stages of development. Representative images of hematoxylin and eosin stains and quantification of airspace density using the radial airspace count (RAC) and parenchymal to airspace ratio using the mean linear intercept (MLI), in A, canalicular and B, saccular stages of lung development. Scale bar $=50 \mu \mathrm{m}$. Gene expression levels of factors involved in branching morphogenesis in control and nitrofenexposed untreated and AFSC-EV treated lung explants at the C, canalicular and D, saccular stages of lung development. Data were analyzed using Kruskal-Wallis test for E17.5 MLI and RAC and E20.5 RAC (A,B) and one-way ANOVA using Tukey's multiple comparison test for all other analyses (A-D). Data are presented as mean \pm SD $(n=$ at least 4 biological replicates per group for RAC and MLI; $\mathrm{n}=3$ biological replicates per group for gene expression analysis). Only significant differences $(\mathrm{p}<0.05)$ are reported in the graphs.

Figure 2. AFSC-EV administration promotes differentiation of alveolar lung epithelial cells at the canalicular stage of fetal lung development. Markers of AT1 (PDPN) and AT2 (SPC) were analyzed at the A, gene (RT-qPCR), and B, protein (Western blot) expression level. ACTB was used as a loading control. C, Representative immunofluorescence images of PDPN (red) and SPC (green) co-localization with the epithelial marker E-cadherin (ECAD). DAPI was used as a nuclear stain. Scale bar $=50 \mu \mathrm{m}$. Data were analyzed using one-way ANOVA using Tukey's multiple comparison test. Data are presented as mean \pm SD ( $n=$ at least 3 biological replicates per 
group for gene and protein expression). Only significant differences $(\mathrm{p}<0.05)$ are reported in the graphs.

Figure 3. AFSC-EV administration promotes differentiation of alveolar and bronchial lung epithelial cells at the saccular stage of fetal lung development. Markers of AT1 (PDPN), AT2 (SPC), club (CC10), basal (P63), ciliated epithelial (FOXJ1) and pulmonary neuroendocrine (ASCL1) were analyzed at the A, gene (RT-qPCR), and B, protein (Western blot) expression level. ACTB was used as a loading control. C, Representative immunofluorescence images of PDPN (red) and SPC (green) co-localization with the epithelial marker ECAD and ASCL1, CC10, FOXJ1 (green) immunolocalization in all fetal lung groups. DAPI was used as a nuclear stain. Scale bar $=50 \mu \mathrm{m}$. Data were analyzed using one-way ANOVA using Tukey's multiple comparison test. Data are presented as mean $\pm \mathrm{SD}(\mathrm{n}=$ at least 3 biological replicates per group for gene and protein expression). Only significant differences $(\mathrm{p}<0.05)$ are reported in the graphs.

Figure 4. AFSC-EV administration has regenerative effects on the fetal lung mesenchyme. A, Mesenchymal markers for fibroblast (Pdgfra), lipofibroblast (Pparg, Thy1) and myofibroblast (Eln, Acta2) cells were analyzed in primary fibroblasts isolated from fetal lungs. Mesenchymal markers analyzed at the B, (RT-qPCR), and B, protein (Western blot) expression levels at the canalicular stage. ACTB was used as a loading control. D, Representative immunofluorescence images of the increased PDGFRA+ fibroblasts and diminished lipofibroblast cells at the canalicular stage. DAPI was used as a nuclear stain. Scale bar $=50 \mu \mathrm{m}$. These same markers 
were also analyzed at the E, gene and F, protein expression levels at the saccular stages. G, Representative immunofluorescence images of decreased PDGFRA+ fibroblast and lipofibroblast cells at the saccular stage. DAPI was used as a nuclear stain. Scale bar $=50 \mu \mathrm{m}$. Data were analyzed using one-way ANOVA using Tukey's multiple comparison test. Data are presented as mean $\pm \mathrm{SD}$ ( $\mathrm{n}=$ at least 3 biological replicates per group for gene and protein expression). Only significant differences $(\mathrm{p}<0.05)$ are reported in the graphs.

Figure 5. AFSC-EVs modulate fetal lung development and maturation in part through their release of RNA species. Representative images of tracking studies using fluorescently labelled AFSC-EVs (red) in A, E17.5 and B, E20.5 fetal lung explants. DAPI was used as a nuclear stain. C, Network analysis of down-regulated genes responsible for branching morphogenesis and cell differentiation following AFSC-EV treatment (Robo1, Fgfr2, Ascl1, and Pdgfra) with AFSC-EV cargo miRNA. Scale bar $=50 \mu \mathrm{m}$.

\section{Graphical abstract}

Babies with congenital diaphragmatic hernia have hypoplastic lungs characterized by impaired branching morphogenesis and undifferentiated epithelium and mesenchyme. The authors demonstrate that amniotic fluid stem cell extracellular vesicles (AFSC-EVs) administered to rat hypoplastic fetal lungs restore branching and exert regenerative effects on epithelial and mesenchymal cells, partly through miRNA cargo transfer. AFSC-EV beneficial effects were obtained at translationally relevant timepoints. 
Table 1. mRNAs assessed by Real-Time PCR.

\begin{tabular}{|c|c|c|c|}
\hline $\begin{array}{c}\text { Gene } \\
\text { Name }\end{array}$ & Primer & $\begin{array}{c}\text { Product } \\
\text { Size } \\
\text { (bp) }\end{array}$ & $\begin{array}{c}\text { GenBank } \\
\text { Accession } \\
\text { Number }\end{array}$ \\
\hline \multirow{2}{*}{$F g f 10$} & for: 5'-AGCTGTTCTCCTTCACCAAGTA-3' & \multirow{2}{*}{118} & \multirow{2}{*}{ NM_012951.1 } \\
\hline & rev: 5'-ACTCCGATTTCCACTGATGTTA-3' & & \\
\hline \multirow{2}{*}{ Fgfr2 } & for: 5'-TGCCTGGTGGAGAATGAATAC-3' & \multirow{2}{*}{168} & \multirow{2}{*}{ NM_012712.1 } \\
\hline & rev: 5'-GGGCTGGGCATCACTATAA-3' & & \\
\hline \multirow{2}{*}{ Robol } & for: 5'-GCGAATCGGAGTGGTTAGTT-3' & \multirow{2}{*}{101} & \multirow{2}{*}{ NM_022188.2 } \\
\hline & rev: 5'-GCGGGCCTTAATCTCGTAGT-3' & & \\
\hline \multirow{2}{*}{ Robo2 } & for: 5'-TGCAACCATTCGCTCTGTAGT-3' & \multirow{2}{*}{223} & \multirow{2}{*}{ NM_032106.3 } \\
\hline & rev: 5'-ACCACCGATGCCAGCTATAA-3' & & \\
\hline \multirow{2}{*}{ Slit2 } & for: 5'-GAAGCAAGAGGCGGAAATACT-3' & \multirow{2}{*}{163} & \multirow{2}{*}{ NM_022632.2 } \\
\hline & rev: 5'-TCCAACATGGTCAAGAAGTGTA-3' & & \\
\hline \multirow{2}{*}{ Ephb2 } & for: 5'-AACGGTGTGATCCTGGACTAT-3' & \multirow{2}{*}{229} & \multirow{2}{*}{ DQ011670.1 } \\
\hline & rev: 5'-CCTTGATGCTGGTCTGGTACT-3' & & \\
\hline \multirow{2}{*}{ Efnb2 } & for: 5'-GTGGTGCTGCTGCTCAAGTA-3' & \multirow{2}{*}{192} & \multirow{2}{*}{ NM_001107328.2 } \\
\hline & rev: 5'-CCCGCTGACCTTCTCGTAGT-3' & & \\
\hline \multirow{2}{*}{ Nrpl } & for: 5'-TGGTGAGCCCTGTGGTCTA -3' & \multirow{2}{*}{123} & \multirow{2}{*}{ NM_145098.2 } \\
\hline & rev: 5'-CATATTCCTCTGGCTTCTGGTAGT-3' & & \\
\hline \multirow{2}{*}{ Ctnnbl } & for: 5'-CGCCAGTGGATTCCGTACT-3' & \multirow{2}{*}{197} & \multirow{2}{*}{ NM_053357.2 } \\
\hline & rev: 5'-GCTTTCCTGATTGCCGTAAG-3' & & \\
\hline \multirow{2}{*}{ Wnt3a } & for: 5'-GTGCAAATGCCACGGACTAT-3' & \multirow{2}{*}{198} & \multirow{2}{*}{ NM_001107005.2 } \\
\hline & rev: 5'-TCTGTGGGCACCTTGAAGTAT-3' & & \\
\hline \multirow{2}{*}{$W n t 5 a$} & for: 5'-AGCAGGTCGCAGGACAGTAT-3' & \multirow{2}{*}{247} & \multirow{2}{*}{ NM_022631.3 } \\
\hline & rev: 5'-CGGACTTGGGTCGATGTAGA-3' & & \\
\hline Sema3a & for: 5'-CGCACAAGACGACAGGATATAA-3' & 142 & NM_017310.1 \\
\hline
\end{tabular}




\begin{tabular}{|c|c|c|c|}
\hline & rev: 5'-TGCATTCCAAGAATGTGCTACT-3' & & \\
\hline \multirow{2}{*}{ Plxna } & for: 5'-TGAACACGCTGGCTCACTAT-3' & \multirow{2}{*}{133} & \multirow{2}{*}{ XM_002729398.6 } \\
\hline & rev: 5'-CGCAGCATGCTCTCGTATC-3' & & \\
\hline \multirow{2}{*}{$P d p n$} & for: 5'-CCTCCACTTGCCAGCAGTA-3' & \multirow{2}{*}{117} & \multirow{2}{*}{ NM_019358.1 } \\
\hline & rev: 5'-GCATGTGGTCCTCAATCATAAC-3' & & \\
\hline \multirow{2}{*}{ Sftpc } & for: 5'-AACGCCTTCTCATCGTGGTT-3' & \multirow{2}{*}{247} & \multirow{2}{*}{ NM_017342.2 } \\
\hline & rev: 5'-GGCTTATAGGCGGTCAGGAG-3' & & \\
\hline \multirow{2}{*}{$\begin{array}{c}\text { Scgblal/ } \\
\text { Cc10 }\end{array}$} & for: 5'-ATACCCTCCCACAGGAGACC-3' & \multirow{2}{*}{153} & \multirow{2}{*}{ NM_013051.1 } \\
\hline & rev: 5'-AGGAGGGAGAGGGGAATGAC-3' & & \\
\hline \multirow{2}{*}{ Tp63 } & for: 5'-CATCAAAGCTCCATTGCTGTA-3' & \multirow{2}{*}{107} & \multirow{2}{*}{ NM_001127343.1 } \\
\hline & rev: 5'-CTTTGCCCTGGTTTTGTAGAC -3' & & \\
\hline \multirow{2}{*}{ Foxj1 } & for: 5'-GTGAGCTGGAACCCCTCAAA-3' & \multirow{2}{*}{238} & \multirow{2}{*}{ NM_053832.2 } \\
\hline & rev: 5'-GTGGCCAGGAAGGTCTCATC-3' & & \\
\hline \multirow{2}{*}{ Ascll } & for: 5'-GGCTCCAACAGCGTCTCTAC-3' & \multirow{2}{*}{169} & \multirow{2}{*}{ NM_022384.2 } \\
\hline & rev: 5'-AGCGGTTGTCTGAGAATGAATA-3' & & \\
\hline \multirow{2}{*}{ Pdgfra } & for: 5'-TTCCTCCGGGCTATCGGATT -3' & \multirow{2}{*}{270} & \multirow{2}{*}{ NM_012802.1 } \\
\hline & rev: 5'-ATTTCCACGTTGGGGTCCTC-3' & & \\
\hline \multirow{2}{*}{ Pparg } & for: 5'-GGTGAAACTCTGGGAGATCCT-3' & \multirow{2}{*}{145} & \multirow{2}{*}{ NM_013124.3 } \\
\hline & rev: 5'-GGTCCACAGAGCTGATTCCG-3' & & \\
\hline \multirow{2}{*}{ Thyl } & for: 5'-CAAGAGGAGGCTGCAAGCTA-3' & \multirow{2}{*}{217} & \multirow{2}{*}{ NM_012673.2 } \\
\hline & rev: 5'-CAGGCTGAACTCATGCTGGA-3' & & \\
\hline \multirow{2}{*}{$E \ln$} & for: 5'-AGCAAGACCTGGGTTTGGAC-3' & \multirow{2}{*}{162} & \multirow{2}{*}{ NM_012722.1 } \\
\hline & rev: 5'- CACTTTCTCTTCCGGCCACA-3' & & \\
\hline \multirow{2}{*}{ Acta2 } & for: 5'-GCTGCTCCAGCTATGTGTGA-3' & & \\
\hline & rev: 5'-AGTTGGTGATGATGCCGTGT-3' & $2 J 3$ & 1VIV_OJIVO4.2 \\
\hline & for: 5'-GTCCACCCGCGAGTACAAC-3' & & \\
\hline Actb & rev: 5'-GGATGCCTCTCTTGCTCTGG-3' & 260 & NM_031144.3 \\
\hline
\end{tabular}


Table 2. Antibodies utilized for imaging and Western blotting.

\begin{tabular}{|c|c|c|c|c|}
\hline Antibody & $\begin{array}{c}\text { Antibody } \\
\text { host }\end{array}$ & Manufacturer/Catalogue \# & Application & Dilution Factor \\
\hline PDPN & Rabbit & ThermoFisher/P5374 & $\mathrm{WB}, \mathrm{IF}$ & 1:200 (IF), 1:1000 (WB) \\
\hline EPCAM & Rabbit & Abcam/ab213500 & IF & $1: 100$ \\
\hline SPC & Rabbit & Abcam/ab90716 & $\mathrm{WB}, \mathrm{IF}$ & $1: 400(\mathrm{IF}), 1: 1000(\mathrm{WB})$ \\
\hline ECAD & Mouse & Abcam/ab231303 & IF & $1: 100$ \\
\hline CC10 & Mouse & Santa-Cruz/Sc-3659921 & WB, IF & $1: 100(\mathrm{IF}), 1: 1000(\mathrm{WB})$ \\
\hline FOXJ1 & Mouse & Santa-Cruz/Sc-53139 & $\mathrm{WB}, \mathrm{IF}$ & 1:50 (IF), 1:500 (WB) \\
\hline P63 & Rabbit & Abcam/ab124762 & WB, IF & $1: 50$ (IF), 1:500 (WB) \\
\hline ASCL1 & Rabbit & Invitrogen/14579482 & WB,IF & 1:250 (IF), 1:1000 (WB) \\
\hline PPARG & Rabbit & ThermoFisher/PA3 821A & WB, IF & 1:500 (IF), 1:1000 (WB) \\
\hline PDGFRA & Goat & R\&D Systems/AF1062 & WB, IF & 1:500 (IF), 1:1000 (WB) \\
\hline $\begin{array}{l}\text { IgG Alexafluor- } \\
488\end{array}$ & Rabbit & $\mathrm{CST} / 4412 \mathrm{~S}$ & IF & $1: 1000$ \\
\hline $\begin{array}{l}\text { IgG Alexafluor- } \\
555\end{array}$ & Mouse & CST/4409 & IF & $1: 1000$ \\
\hline $\begin{array}{l}\text { IgG AlexaFluor- } \\
488\end{array}$ & Goat & Invitrogen/A11055 & IF & $1: 1000$ \\
\hline IgG HRP & Mouse & CST/7076S & WB & $1: 1000$ \\
\hline IgG HRP & Rabbit & CST/7074 & WB & $1: 1000$ \\
\hline IgG HRP & Goat & Invitrogen/31402 & WB & $1: 1000$ \\
\hline
\end{tabular}

CST: Cell signaling technology

WB: Western blot

IF: Immunofluorescence 
RAC

Mㄴ
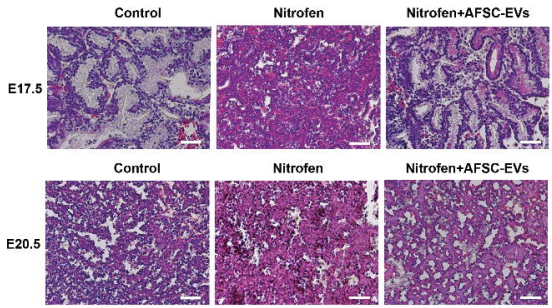

Nitrofen

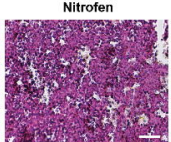

Nitrofen+AFSC-EVs

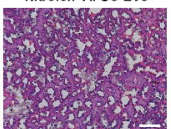

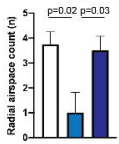

RAC

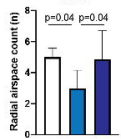



MLI

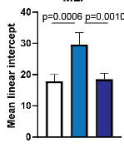

Legend

口 Control

$\square$ Niirufen

$\square$ Nitroren+AFSC-EVs
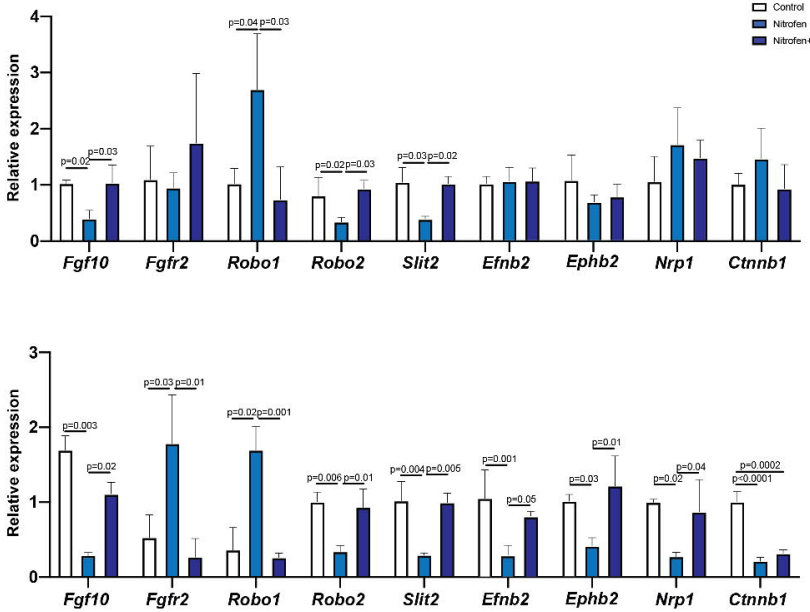

Figure 1 

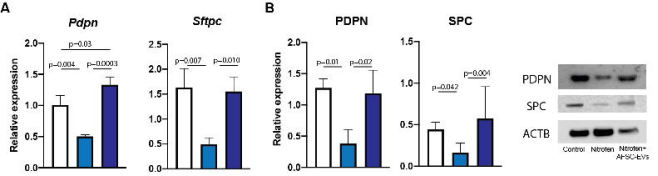

$\square$ Control

$\square$ Nitroien

$\square$ Nitroien+AFSC-EVa

C

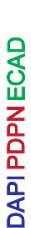



()

م

()

$\frac{8}{4}$

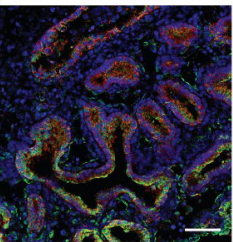

Nitrofen
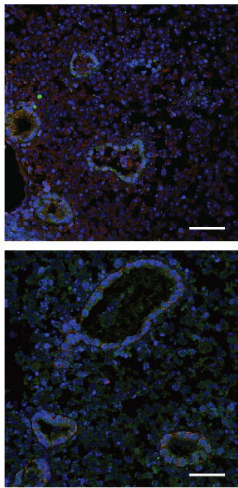

Nitrofen+AFSC-EVs
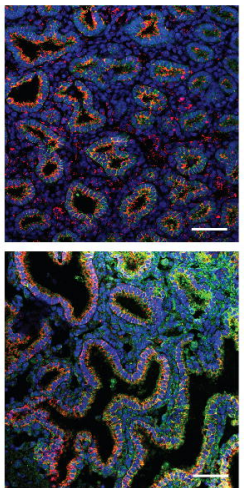

Figure 2 


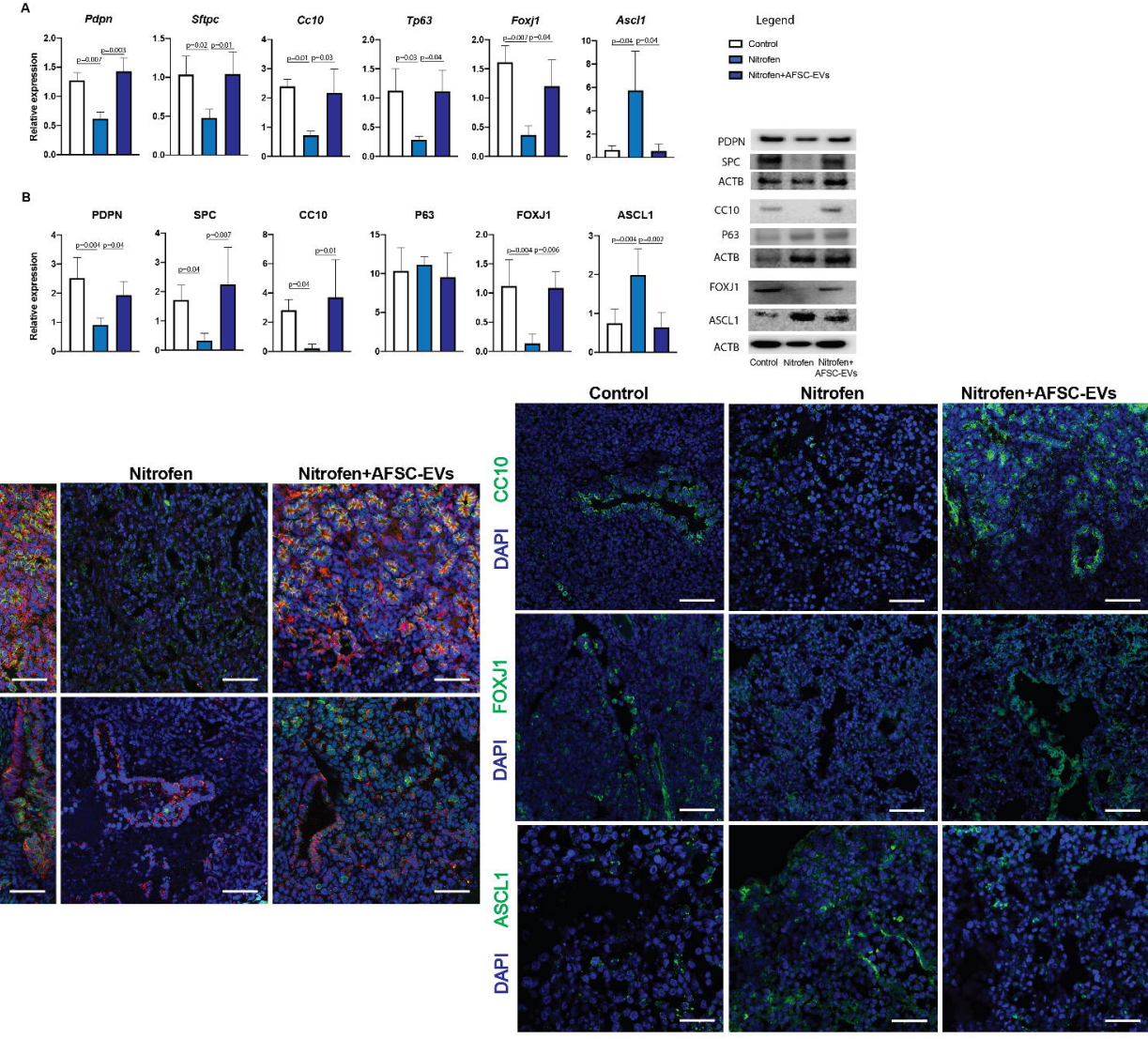




\section{E17.5}
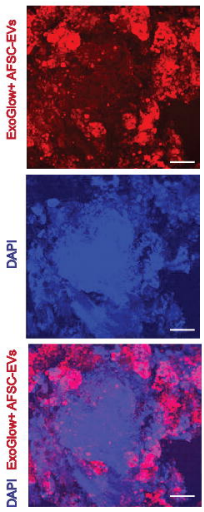

E20.5


\section{。}



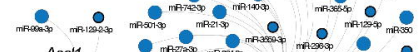

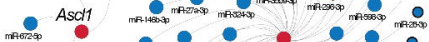

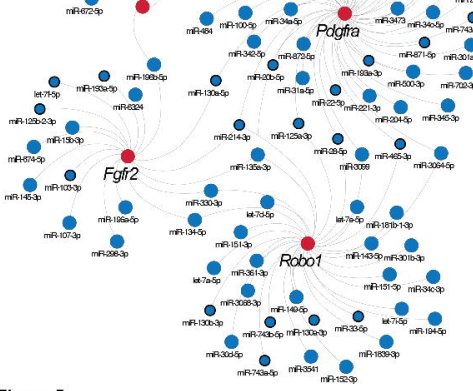

Figure 5 


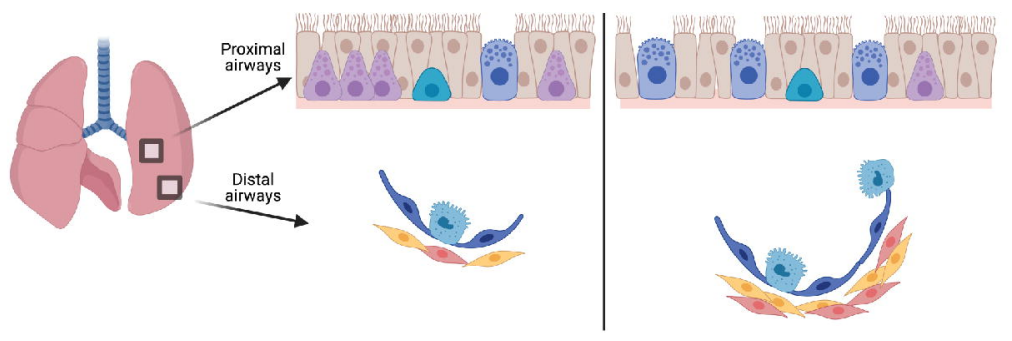

\section{Legend}

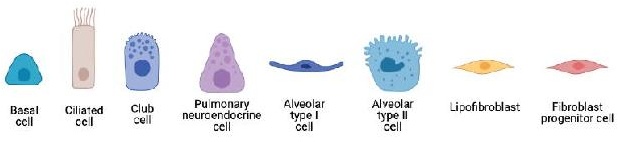

